\title{
A Real-Time Temperature Data Transmission Approach for Intelligent Cooling Control of Mass Concrete
}

\author{
Peng Lin, Qingbin Li, and Pinyu Jia \\ State Key Laboratory of Hydroscience and Engineering, Tsinghua University, Beijing 100084, China \\ Correspondence should be addressed to Peng Lin; celinpe@tsinghua.edu.cn
}

Received 28 December 2013; Accepted 7 February 2014; Published 12 March 2014

Academic Editor: Ting-Hua Yi

Copyright (c) 2014 Peng Lin et al. This is an open access article distributed under the Creative Commons Attribution License, which permits unrestricted use, distribution, and reproduction in any medium, provided the original work is properly cited.

\begin{abstract}
The primary aim of the study presented in this paper is to propose a real-time temperature data transmission approach for intelligent cooling control of mass concrete. A mathematical description of a digital temperature control model is introduced in detail. Based on pipe mounted and electrically linked temperature sensors, together with postdata handling hardware and software, a stable, real-time, highly effective temperature data transmission solution technique is developed and utilized within the intelligent mass concrete cooling control system. Once the user has issued the relevant command, the proposed programmable logic controllers (PLC) code performs all necessary steps without further interaction. The code can control the hardware, obtain, read, and perform calculations, and display the data accurately. Hardening concrete is an aggregate of complex physicochemical processes including the liberation of heat. The proposed control system prevented unwanted structural change within the massive concrete blocks caused by these exothermic processes based on an application case study analysis. In conclusion, the proposed temperature data transmission approach has proved very useful for the temperature monitoring of a high arch dam and is able to control thermal stresses in mass concrete for similar projects involving mass concrete.
\end{abstract}

\section{Introduction}

In hydraulic engineering, due to the poor conductivity of concrete, high temperature gradients may occur between the interior and the surface of the structural elements or between parts of an element due to processes of sequential concreting in phases $[1,2]$. The temperature gradients may easily cause tensile stresses, which exceed the tensile strength and result in the cracking of early age mass concrete $[3,4]$. Much engineering practice has showed that it is difficult to control its highest temperature within an allowable range when pouring concrete in the hot season. This is because pouring concrete is affected by the pouring temperature, solar radiation, and cooling pipes. As the thermal control of mass concrete is a common problem in civil engineering, sensor technologies have developed quickly and are much utilized in structural health monitoring in civil and hydraulic engineering [5-9]. Accurate real-time field control must be achieved.

Data communication plays an important role in the rapid development of intelligent cooling control systems. Intelligent cooling control systems [1, 10-12] enable units of concrete poured during dam construction to cool appropriately.
Making data communication possible between all cooling control information from all field control systems is also provided to top-level management. The current temperature digital data collection methods and the control exercised with the use of manual records are very rudimentary due to poor accuracy and lack of data reliability $[10,13]$. The main factors supporting accurate temperature control are real-time measurements, accurate collection in real time of dam internal temperature data, cooling pipe inlet and outlet water temperatures, and weather temperature data. Thus, the development of a real-time temperature data transmission approach for intelligent cooling control of mass concrete is of important academic and engineering significance.

Digital computers were first applied to industrial process control in the late 1950s [14]. The individual temperature, pressure, flow, and the like and feedback loops were locally controlled by electronic or pneumatic analog controllers. Each individual loop operated can optimize the overall operation. In 1962, imperial chemical industries made a drastic departure from this approach and a direct digital control (DDC) was coined to emphasize that the computer controlled 
the process directly. In DDC systems, analog controllers were no longer used. In 1970s, microcomputers have already made a great impact on the process control field. They are replacing analog hardware even as single loop controllers [15]. Multiple loop single computer systems have variability interface and software design. The distributed computer control system (DCCS) was developed, which is facilitated by adopting a multilevel or hierarchical viewpoint of control strategy. On the lowest level of control, main tasks handled acquisition of process data and collection of data. For complex process plant monitoring, supervisory control and data acquisition (SCADA) systems are available. The basic functions of SCADA system include data acquisition and communication, events and alarms reporting, data processing, and partial process control. The other main and early application area of digital methods was machine tool numerical control, which developed at about the same time as computer control in process industries, for example, numerically controlled (NC), computerized numerical control (CNC), and direct numerical control (DNC). One of the most ingenious devices ever devised to advance the field of industrial automation is the PLC. The PLC, a microprocessor-based general-purpose device, provides a "menu" of basic operations that can be configured by programming to create logic control system for any application [16]. Thus, the recent appearance of powerful and inexpensive microcomputers has made digital control practical for a wide variety of applications. In fact, now every process is a candidate for digital control [17].

Digital data transmission technologies have attracted the attention of many researchers and engineers in recent years [18-27]. For example, Italiano et al. [19] developed the SMM, sea-floor monitoring module, for real-time data transmission from the sea bottom, equipped with multidisciplinary sensors. Bayindir and Cetinceviz [20] describe a water pumping control system designed for production plant and implemented experimentally in a laboratory. An intelligent system for distributed control of an anaerobic wastewater treatment process was developed by Flores et al. [21]. Language of temporal ordering specification (LOTOS) was used in the specification of industrial bus communication protocols [22]. Communication structures have been used for sensory data in mobile robots [23], and the network is used as a bus system for long discharge data acquisition and data processing [24]. The timing behaviour of real-time stations in legacy bus based industrial Ethernet networks has been enforced [25]. A study on real-time sensor signal transmission based on polynomial predictive filtering technology has also been carried out by Dong et al. [27]. Connecting PLC to control and data acquisition enabled a comparison of the Joint European Torus (JET) and Wendelstein 7-X approaches to be made [26]. These studies have demonstrated convenient solutions, reliable operation, and robust and flexible construction suitable for industrial applications. However, few studies were concerned with the cooling control of mass concrete in a dam under complex electromagnetic environment, especially the problem of the large quantity of temperature digital data to be transmitted in relation to temperature control during concrete block pouring.

This paper, relating to a real-time temperature data transmission approach at a super high arch dam site, presents a detailed description of certain aspects of a control system that is intended to ensure the structural integrity of massive concrete structures during the construction process. The cooling control system prevents unwanted structural changes within massive concrete blocks by carrying away the heat generated by the exothermic processes of cement hydration. The proposed temperature transmission approach collects temperature data from many sensors and prevents the hardening concrete from overheating by controlling the water flow inside the cooling pipes constructed within the concrete structure. The temperature transmission system includes the acquisition of temperature data, management of various intelligent modules, temperature data handling, sorting and filtering, and temperature data transfer to the module control server. The detailed PLC software configuration and programming are also illustrated. An application case of temperature monitoring of the Xiluodu arch dam during the construction period is discussed in detail. The paper aims to provide a convenient solution to the process of cooling control via the pipes in a situation where the laying of cables is not possible.

\section{Temperature Digital Data Transmission Approach}

2.1. Mathematical Description of Digital Temperature Control Model. In this study, each basic poured block temperature control unit has a fixed structure (see Figure 1): one electric solenoid proportional valve, one outlet water flow transmitter and two or more temperature sensors installed at the water pipe inlet and outlet, adjacent concrete temperature, and so forth. The mass concrete temperature control kernel collected all the data and commanded the execution of individual control actions indirectly via the PLC system, which was easily set up to handle the field bus.

In this study, a prerequisite signal processing technique was used $[10,11]$ as the proposed closed-loop digital control system for mass concrete cooling on a construction site. The digital control system with analog sensors (flow sensor, thermometer) included an analog prefilter placed between the sensor and the sampler $(\mathrm{A} / \mathrm{D}$ converter) as antialiasing device, shown in Figure 2. The prefilters are low-pass, and the simplest transfer function is used:

$$
H_{\mathrm{pf}}(s)=\frac{a}{s+a}
$$

so that the noise above the prefilter breakpoint $a$ was attenuated. The design goal is to provide enough attenuation at half the sample rate $\left(\omega_{s} / 2\right)$ so that the noise above $\omega_{s} / 2$, when aliased into lower frequencies by the sampler, will not be detrimental to the control-system performance. 


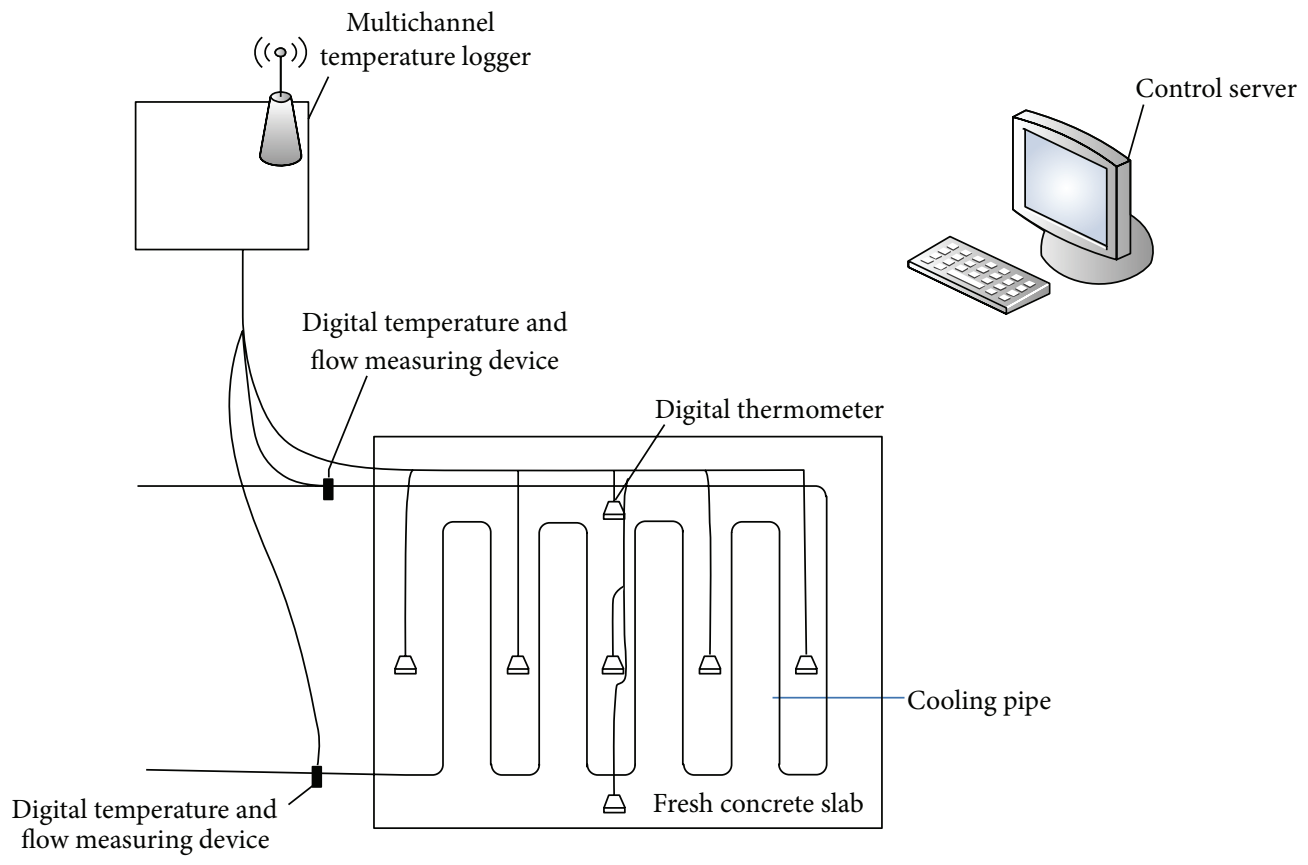

FIGURE 1: The schematic diagram of temperature and flow acquisition, collecting, and analysing system.

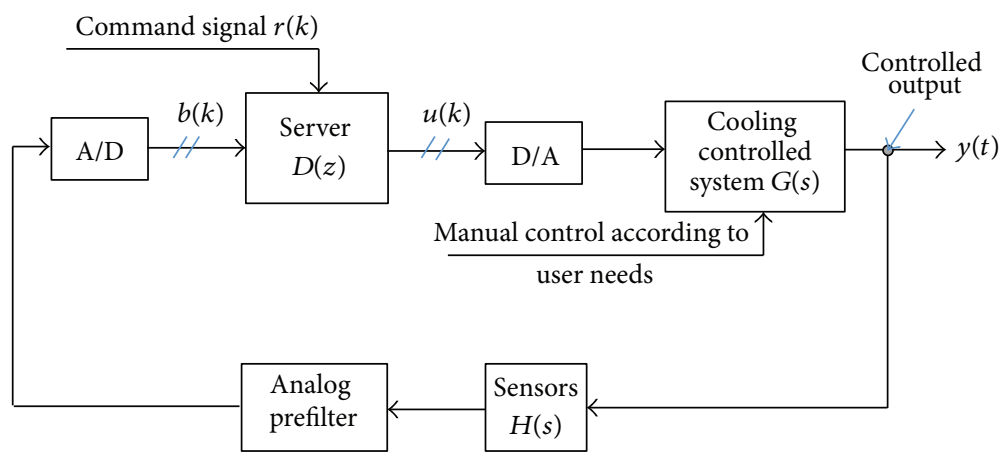

FIGURE 2: Block diagram for control process.

For this cooling control system, the basic configuration and block diagram are shown in Figure 2, where

$G(s)=$ transfer function of the controlled system (continuous-time system);

$H(s)=$ transfer function of the analogue sensor;

$D(z)=$ transfer function of the digital control algorithm.

The analogue and digital parts of the system are connected through $\mathrm{D} / \mathrm{A}$ and $\mathrm{A} / \mathrm{D}$ converters. The server with its internal clock drives the $\mathrm{D} / \mathrm{A}$ and $\mathrm{A} / \mathrm{D}$ converters. It compares the command signal $r(k)$ with the feedback signal $b(k)$ and generates the control signal $u(k)$ to be sent to the final control elements of the cooling control system. These signals are computed fusing the digital control algorithm $D(z)$, stored in the memory of the compute server.

To suit the complications of a real construction site, the field bus is designed to link the sensors and actuators: the Profibus network links all the analogue signal input and output modules which are cyclically converting the A/D or D/A signals. Mathematical models generally are used to represent the digital control devices and the system. The A/D converter model [14] used in this system is shown in Figure 3(a). For the field temperature control process, a continuous-time function $f(t), t \geq 0$, is the input and the sequence of real numbers $f(k), k=0,1,2, \ldots$, is the output. The following relationship holds between input and output:

$$
f(k)=f(t=k T),
$$

where $T$ is the sampler time interval between enquiring flow or temperature and acquiring the result. The sequence $f(k)$ can be treated as a train of impulses represented by the continuous-time function $f^{\prime}(t)$ :

$$
\begin{aligned}
f^{\prime}(t)= & f(0) \delta(t)+f(1) \delta(t-T) \\
& +f(2) \delta(t-2 T)+\cdots=\sum_{k=0}^{\infty} f(k) \delta(t-k T)
\end{aligned}
$$




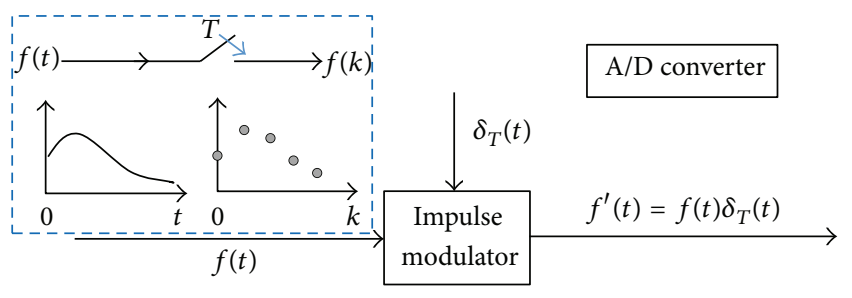

(a)

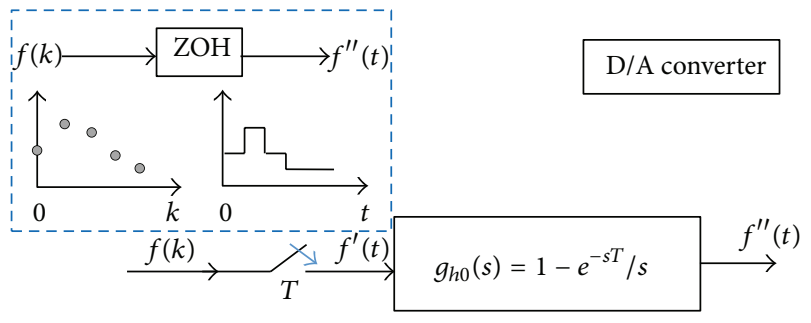

(b)

Figure 3: A model of an A/D or D/A converter.

The model of D/A converter is shown in Figure 3(b). A sequence of numbers $f(k), k=0,1,2, \ldots$, is the input, and the continuous-time function $f^{\prime \prime}(t), t \geq 0$, is the output. The following relationship holds between input and output:

$$
f^{\prime \prime}(t)=f(k) ; \quad k T \leq t<(k+1) T .
$$

Each sample of the sequence $f(k)$ may be treated as an impulse function of the form $f(k) \delta(t-k T)$. The zero-order hold $(\mathrm{ZOH})$ of Figure $3(\mathrm{~b})$ can thus be viewed as a linear time-invariant system that converts the impulse $f(k) \delta(t-k T)$ into a pulse of height $f(k)$ and width $T$. The D/A converter may, therefore, be modelled by Figure 3(b), where the $\mathrm{ZOH}$ is a system whose response to a unit impulse $\delta(t)$ is a unit pulse $g_{h 0}(t)$ of width $T$. The Laplace transform of $g_{h 0}(t)$ is the transfer function of the hold operation; namely,

$$
g_{h 0}(s)=\frac{1-e^{-s T}}{s} .
$$

2.2. Temperature Digital Data Transmission. For dam temperature control system, shown in Figure 1, the temperature sensors sampling time could be as long as one minute. A Profibus network and corresponding modules would dominate the control cost unnecessarily and therefore a lower cost field bus called ITU was engaged. An intelligent module can transfer the ITU signals into RS485 protocol, which most kinds of PLC support. The intelligent temperature module, which has 8 channels, can link a maximum of 256 different ITU sensors usually via three core cables. After initialization, the module can exchange the digital data with PLC basis of RS485 protocol. One RS485 master can communicate with 127 slaves and this means one PLC CPU can acquire up to 32,512 ITU temperature sensors. In this control system, the Siemens Simatic S7-300 PLC was used as the data bridge between the control model computer and the field sensors and actuators. The specified PLC programming language FBD (function block diagram) and standard function library provided the software engineering basic environment. The software tasks were condensed into the three parts of collecting the temperature and flow data, communicating with the model server, and the water flow proportional integral derivative (PID) control.

2.2.1. Data Inquiring and Acquiring. There are a number of commands defined to fetch the status or data from the intelligent temperature module LTM8663 via the RS485 protocol, which are in ASCII format. The most important commands from PLC to the intelligent module are as follows.

(1) *AAN (CR). The command name: to read all the sensors' ID linked to the channel " $N$ " of the specified intelligent module " $A A$ " (ranging from $00 \mathrm{~h}$ to $\mathrm{FF}$ h); here "CR" is the carriage return character (ASCII code " $0 \mathrm{Dh}$ ").

Each ID is preset by a handy tool or PC software to escape the same numbering on the same channel.

(2) \#AAN (CR). The command name: similar to the above "*AAN;" this command tries to read all the sensors' data linked to the channel " $N$ " of the specified intelligent module "AA."

The above two frequently used commands enable PLC to get exactly the total numbers and numbering of linked sensors, as well as their current temperature values.

2.2.2. Data Handling, Sorting, and Filtering. As long as there is a valid response from the intelligent module, the temperature digital data will be first transferred into a data buffer, which is previously defined in the PLC, and then analyzed according to the predefined data format. The standard function block for the PLC will copy the data from the RS485 network to that buffer and generate a pulse indicating that new data is coming; the user program will fetch the channels related data and copy them to corresponding data block according to the current counter.

Since channel data sets are separated, the PLC decides whether they are valid or not on the basis of the received telegram header (here "> " means data are valid). The program will then transfer the raw temperature data into standardized format (floating type). For the specific LTM8663 module used in this system, the original temperature data format is $16 \# \mathrm{~A}$ (byte 1) B (byte 2), and the conversion formula is

$$
t=\frac{[256(B \& 16 \# 7)+A]}{16} .
$$

According to the LTM8663 specification, the RS485 communication protocol is ASCII, and the parameters should be configured in the hardware interface as; the speed transmission rate is $9,600 \mathrm{bps}$, data bits are 8 , stop bits is 1 , and the operating mode is half duplex (RS485) two-wire mode.

After the PLC program has distinguished the total number of sensors of the module, each ID, and actual data, it is not difficult to sort and copy them to the right offset location in the summary data block. Obviously, each sensor ID in one module must be unique. The summary data block is the transmission information to the module control server. 


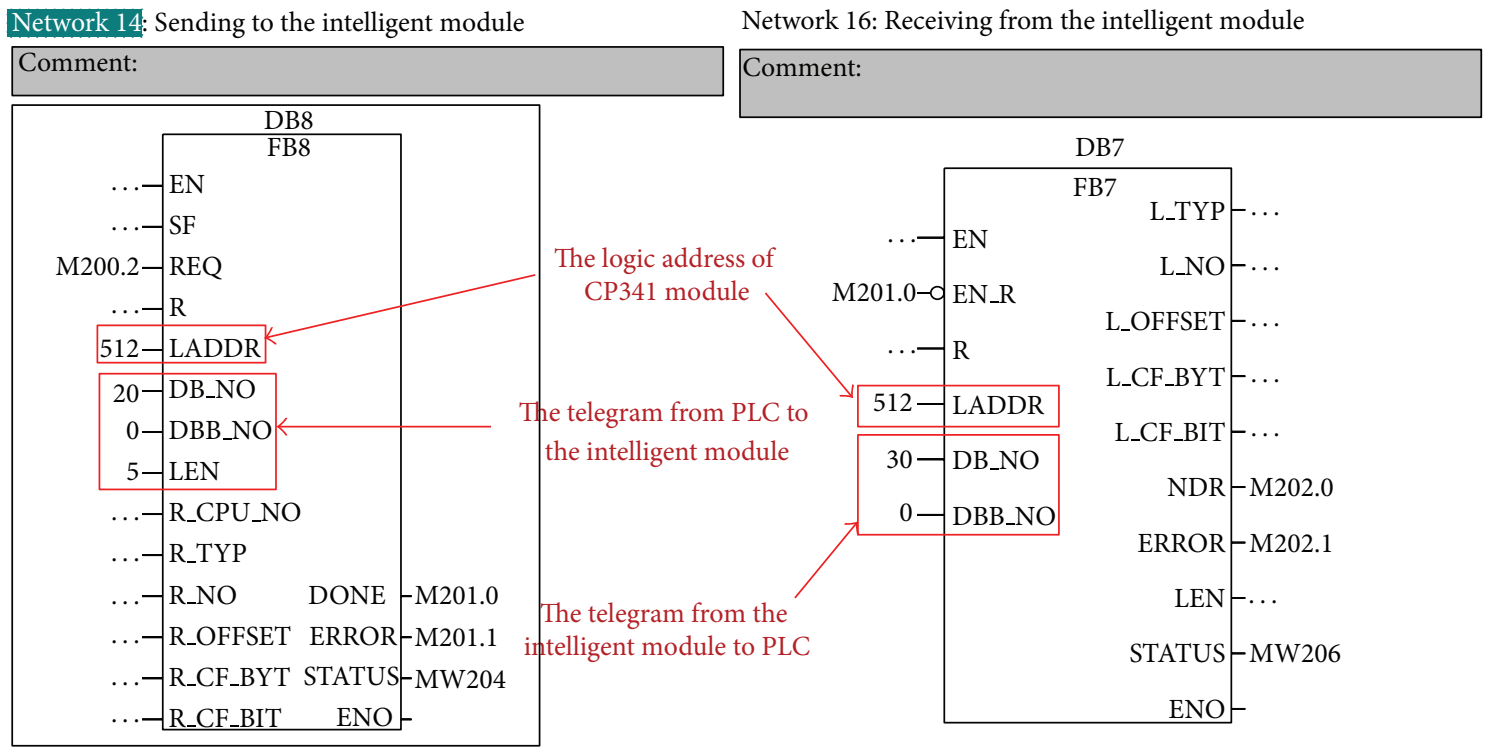

FIGURE 4: Function blocks for communication between CP341 and LTM8663 modules.

2.3. Management of Various Intelligent Modules. On the dam construction site, thousands of sensors were installed on the cooling pipes or cast inside the concrete, enabling various identified intelligent modules to be linked to PLC via RS485 network; PLC software autodecided the polling strategy for the whole network to avoid data acquisition conflicts. To poll each module and channel cyclically, a counter is utilized to differentiate the time frames for each task. In addition, the PLC sends two different commands for each channel since the time interval must be considered. The brief polling sequence is illustrated in [10].

To achieve communication between CP343-1 and the module server, the server must be configured in the Simatic manager. The module server is taken as "another station" here, and in the net two connections should be established for sending and receiving. For the Transmission Control Protocol (TCP) connection; the port addresses of both parties should be fixed.

The real-time data transmission from concrete construction site to the remote module control server was achieved using an industrial Ethernet communication board, with TCP/IP protocol. Depending on the distance, a fibre optic converter or a wireless AP can be used for the data bridge. The standard PLC Ethernet communication function block can easily transfer the data block contents to the target server. The network structure is independent of the sensors. If one or several sensors fail, the master can still read data from other sensors. After replacement, the sensors can be accessed again as the master collects the data on the whole network repeatedly.

\section{PLC Code Configuration}

In this study, the structured control language (SCL) was used as the PLC code. The FBD and the Standard Template Library (STL) included communication with the intelligent module
(RS485), temperature digital data treatment, filtering and sorting, communication with the module server (TCP/IP), and the cooling water PID control.

3.1. Communication with the Intelligent Module LTM8663. The standard function block FB7 and FB8 (P_SND_RK) are designed for RS485 communication structure, shown in Figure 4.

The CP341 module is an extended communication processor which supports RS422 and RS485 protocol. The data block DB20 stores the inquiring command ( 5 bytes) to the intelligent module, and DB30 is the receiving data buffer from the module. The execution of FB7 and FB8 is separated by logic.

3.2. Temperature Digital Data Analysis. When the specific channel temperature digital data is recognized, it is transferred from DB30 to another data block for handling. For example, DB31 handles raw data coming from channel 1, module 0 . As the total number of sensors and each ID is known by the program, each temperature value can be converted into target floating format. The source code below converts the data according to (6).

After the treatment, the sensor actual values are copied to a summary data block (e.g., DB10 for module 0). The offset in the data block will follow the ID. For example, the offset address for the sensor ID 3 is 12 as in Table 1.

3.3. Communication with the Module Server. Two other standard functions (FC50 and FC60) are used for the TCP/IP communication with the module server, as shown in Figure 5. The Ethernet port is integrated in the CPU module. The DB1 is the sending buffer which collects all the sensor information and telegram time stamp, shown in Table 2.

The telegram length is 2022 bytes, including the title, telegram label, length, telegram type, sending time, total circuit 
TABLE 1: The PLC data structure of DB10 for communication with intelligent module.

\begin{tabular}{|c|c|c|c|c|}
\hline Number & Address offset & Name & Data type & Comments \\
\hline 1 & 0 & Module_SN & Integer & Module serial number \\
\hline 2 & 2 & Total_sensors & Real & Total sensors number \\
\hline 3 & 4 & Value_ID1 & Real & SensorValue_ID1 \\
\hline 4 & 8 & Value_ID2 & Real & SensorValue_ID2 \\
\hline 5 & 12 & Value_ID3 & Real & SensorValue_ID3 \\
\hline 6 & 16 & Value_ID4 & Real & SensorValue_ID4 \\
\hline$\vdots$ & $\vdots$ & $\vdots$ & $\vdots$ & $\vdots$ \\
\hline 256 & 1016 & Value_ID254 & Real & SensorValue_ID254 \\
\hline 257 & 1020 & Value_ID255 & Real & SensorValue_ID255 \\
\hline 258 & 1024 & Value_ID256 & Real & SensorValue_ID256 \\
\hline
\end{tabular}

TABLE 2: The PLC data structure of DB1 for sending telegram to module server.

\begin{tabular}{lcccc}
\hline Number & Address offset & Name & Data type & Comments \\
\hline 1 & 0 & Label & String(4) & Fixed as “EDAM" \\
2 & 4 & Length & Integer & Telegram length \\
3 & 6 & Tel_type & INT & Telegram type \\
4 & 8 & SendTime & String(12) & Format: YYYYMMDDHHMM \\
5 & 20 & TotalNumber & INT & Total circuits \\
6 & 22 & Telegraml & UDT1 & User defined data type \\
$\vdots$ & $\vdots$ & $\vdots$ & $\vdots$ & $\vdots$ \\
$n+5$ & $(n-1) * 10+22$ & Telegram $(n)$ & UDT1 & User defined data type \\
$\vdots$ & $\vdots$ & $\vdots$ & $\vdots$ & $\vdots$ \\
\hline
\end{tabular}

TABLE 3: The user defined type (UDT) for each circuit value as a template.

\begin{tabular}{lcccc}
\hline Number & Address offset & Name & Data type & Comments \\
\hline 1 & 0 & Number & Integer & The circuit number \\
2 & 2 & Mode & Byte & Mode, $1=$ auto, $2=$ manual \\
3 & 3 & AlarmWarnning & Byte & $0=$ OK, $1=$ warning, $2=$ alarm \\
4 & 4 & InletTemp & Integer & Water inlet tmperature \\
5 & 6 & OutletTemp & Integer & Water outlet temperature \\
6 & 8 & ActFlow & Integer & Actual water flow \\
\hline
\end{tabular}

numbers, and every item of circuit data. Here a special UDT (user data type, 10 bytes) is defined for each water cooling circuit, including the serial number, auto- or manual mode, status, inlet and outlet temperature, and actual water flow, shown in Table 3. Correspondingly, the DB2 is the receiving buffer containing the commands for each circuit from the module server's control strategy system.

Similarly, the receiving telegram contains the label, length, type, and command for each circuit and the personalized control solution is then realized in this way. Every circuit can have its own cooling water flow target set point or fixed water valve opening output.

\section{Application Case Study}

4.1. Brief Introduction to Dam Site. The proposed method was applied to the temperature control of the Xiluodu arch dam during construction $[28,29]$. The project is located on the Jinshajiang River, in the Leibo County of Sichuan province. The principal structures consist of a double-curvature arch dam 285.5 meters high, crest length about 700 meters, spillway, underground powerhouse, and logway. A total of 6,000,000 cubic meters of concrete were poured. Because of the high stresses in an arch dam structure and the construction process control difficulties, the basic temperature control principle was to maintain ultimately small temperature differences between neighbouring areas of concrete by slow cooling and early cooling. The cooling process should be based on the concrete temperature measurement results taking into consideration the partitioning of the concrete, pouring temperature, water pipes, density of concrete, and ambient temperature. The cooling water flow is adjusted in a timely manner to meet the targeted temperature requirements.

A flexible network structure for field temperature monitoring of the super high arch dam during construction was 


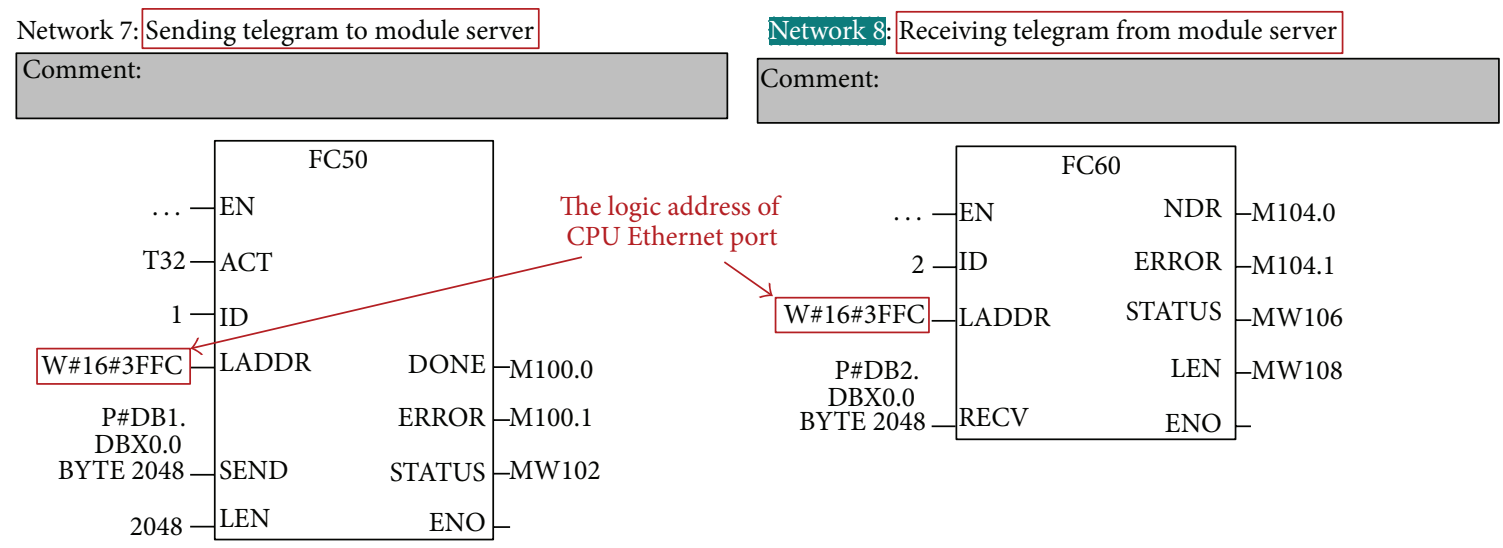

FIgURE 5: The PLC functions for TCP/IP communication with the module server.

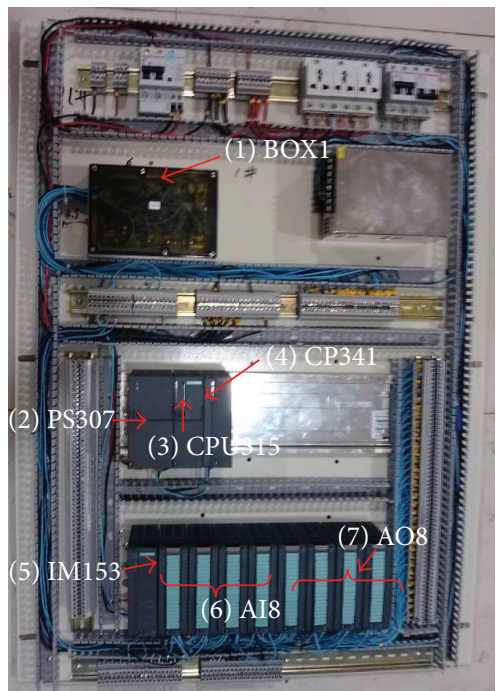

FIgURE 6: The internal layout of the PLC control cubicle.

developed based on the state of the art intelligence control technology [8]. By employing flexible network structure architecture, the bus was also connected to other types of sensors such as dam strain measurement meters, dam compressive stress measurement meters, meters for monitoring joint openings, rock-deformations, and piezometers for seepage flow, all of which were are designed to suit this network specification. Thus, the measurement scope utilized covered a wide spectrum as well as the many concrete temperature measurements sufficient for a super high arch dam.

4.2. Site Control System Arrangement. The control cabinets were prepared in the workshop. Because of the complex electromagnetic environment on the construction site, wiring to the site flow valves and sensors were employed. Figure 6 shows a preassembled cabinet for digital data transmission. Here is the brief description of the main parts of PLC control cubicle.
BOX1: The intelligent temperature module. The circuit connection diagrams for connecting the temperature sensors to the module are shown in Figure 7.

PS307: The Simatic S7-300 PLC power module.

CPU315: The Simatic S7-300 PLC CPU with integrated Profibus and Ethernet interfaces.

CP341: The Simatic RS485 communication processor for the intelligent temperature module.

IM153: The Simatic interface module, which can extend the CPU control to remote areas by Profibus network.

AI8: The Simatic analog input module, which can read 8 analogue signals (current, voltage, resistor, or PT100 sensors). The circuit diagram for connecting the flow meters to the analogue input module is shown in Figure 8. The symbol "SM331" is the analogue input module, which is able to read 8 water flow transducers; please refer to "(6) AI8" of Figure 6.

AO8: The Simatic analogue output module, which can drive 8 analog valves. The circuit diagram for connecting the electric valves to the analogue output module is shown in Figure 9. The symbol "SM332" is the analogue output module, which is able to control 8 proportional valves; please refer to "(7) $\mathrm{AO} 8$ " of Figure 6.

On the dam site, the operators connect the valves, flow meters, and temperature sensors into the PLC cabinets as required according to construction progress.

4.3. Temperature Field Analysis Basis of Real-Time Temperature Data. On the Xiluodu arch dam site, the intelligent cooling control system used modern field bus technology extensively and Siemens PLC devices to comprehensively track the temperature data change at the pouring of every block in real time. Adjustment of the valves and control of water flow were performed using the PID algorithm based on temperature control requirements and key factors such as the highest temperature reached, cooling rate, and temperature 


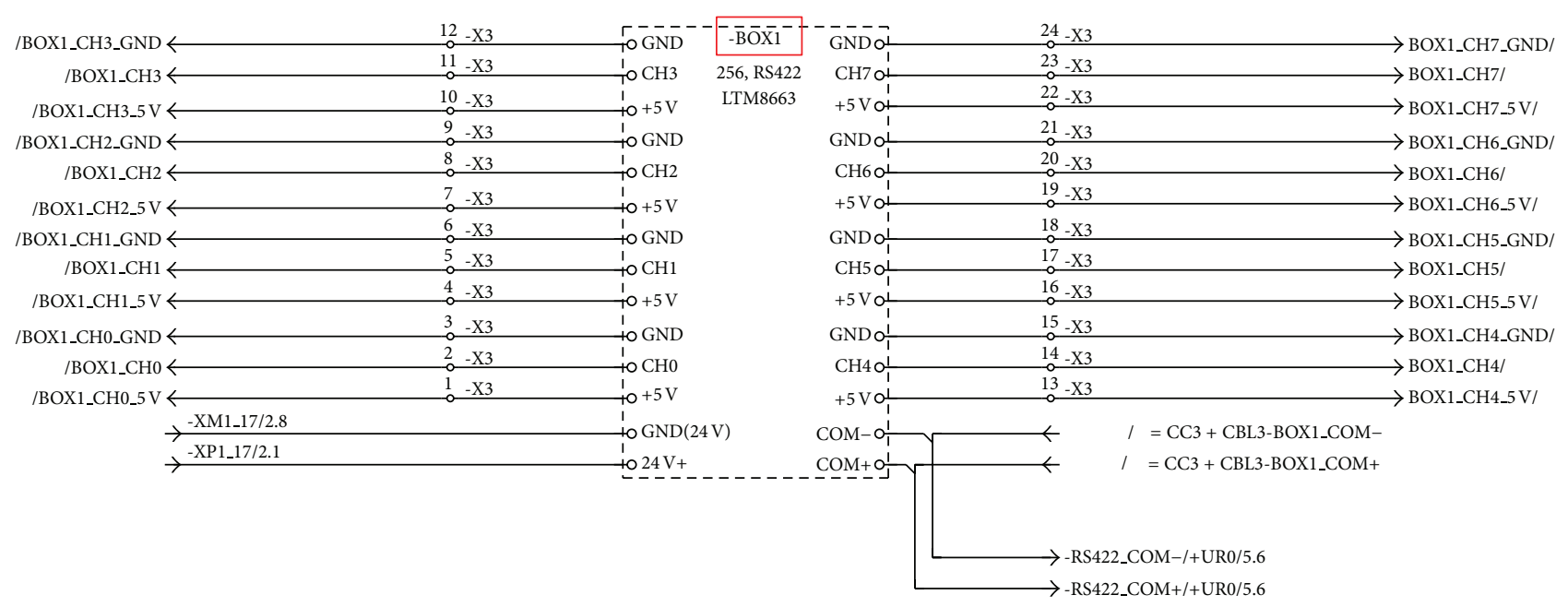

FIgURE 7: The circuit diagram for connecting the temperature sensors to the module.

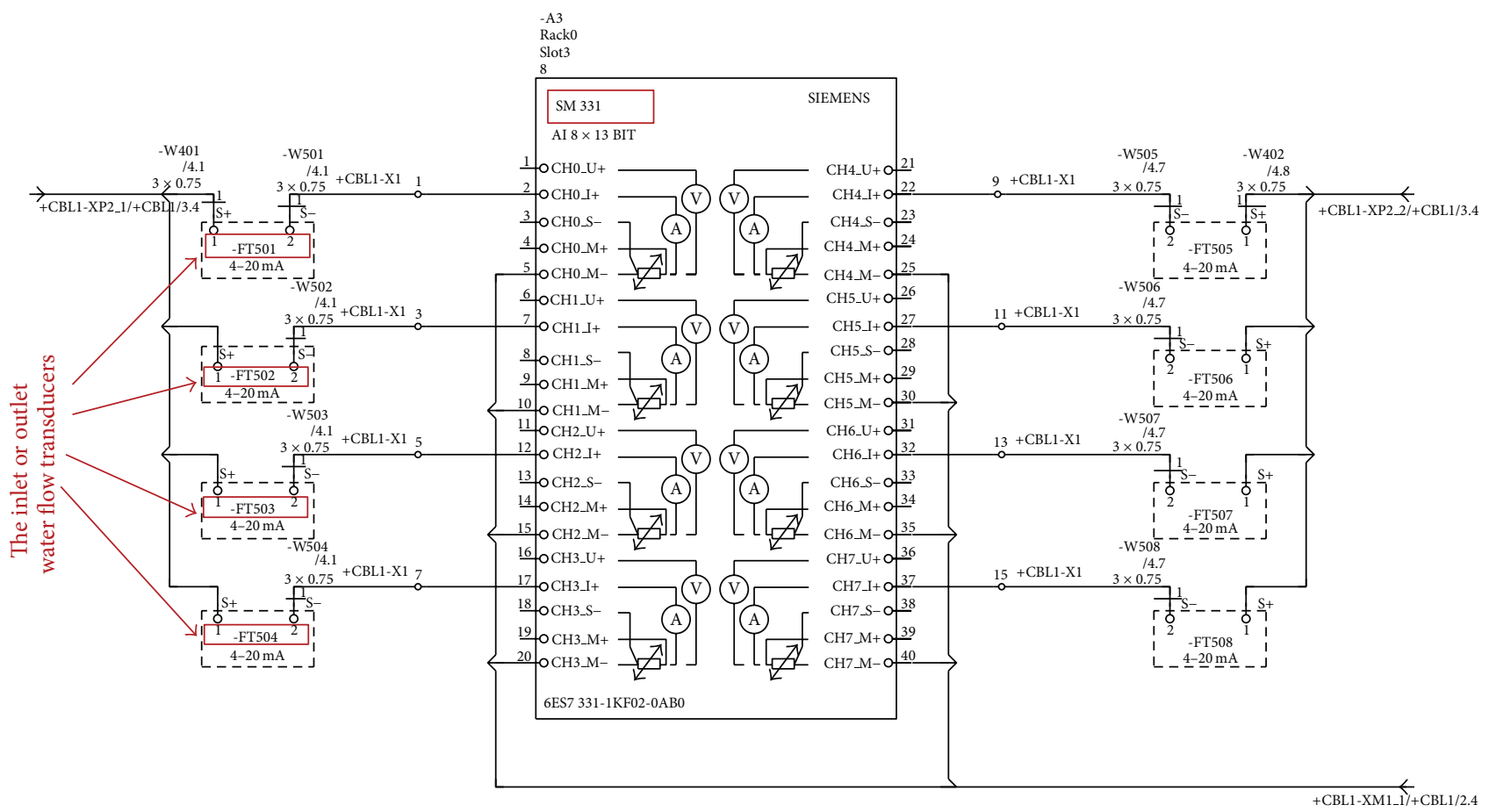

FIGURE 8: The circuit diagram for connecting the temperature sensors to the module.

gradient. A web graphical user interface (GUI) was provided for integrated query, analysis, and prewarning [1].

Based on the proposed temperature data transmission system, the intelligent cooling system overcomes the shortcomings in traditional manually collected data systems, providing an effective dam concrete temperature control and cracking prevention technology both advanced and more intelligent. In particular the advances consist of (1) changing simple manual data collection to real-time data acquisition and feedback, (2) automatically obtaining large quantities of thermodynamic parameters with respect to every block of poured concrete enabling a more customized control function, and (3) enabling a 3D real-time temperature field analysis be carried out, as illustrated in Figure 10. Based on the real-time temperature field analysis, further stress fields can be analyzed to study alternative temperature control plans, not only limited to water flow, but the whole pipework scheme can be adjusted to more optimal configurations.

\section{Conclusions}

This paper presents a real-time temperature data transmission approach for intelligent cooling control of early age mass concrete based on A/D converter model and PLC technology. 


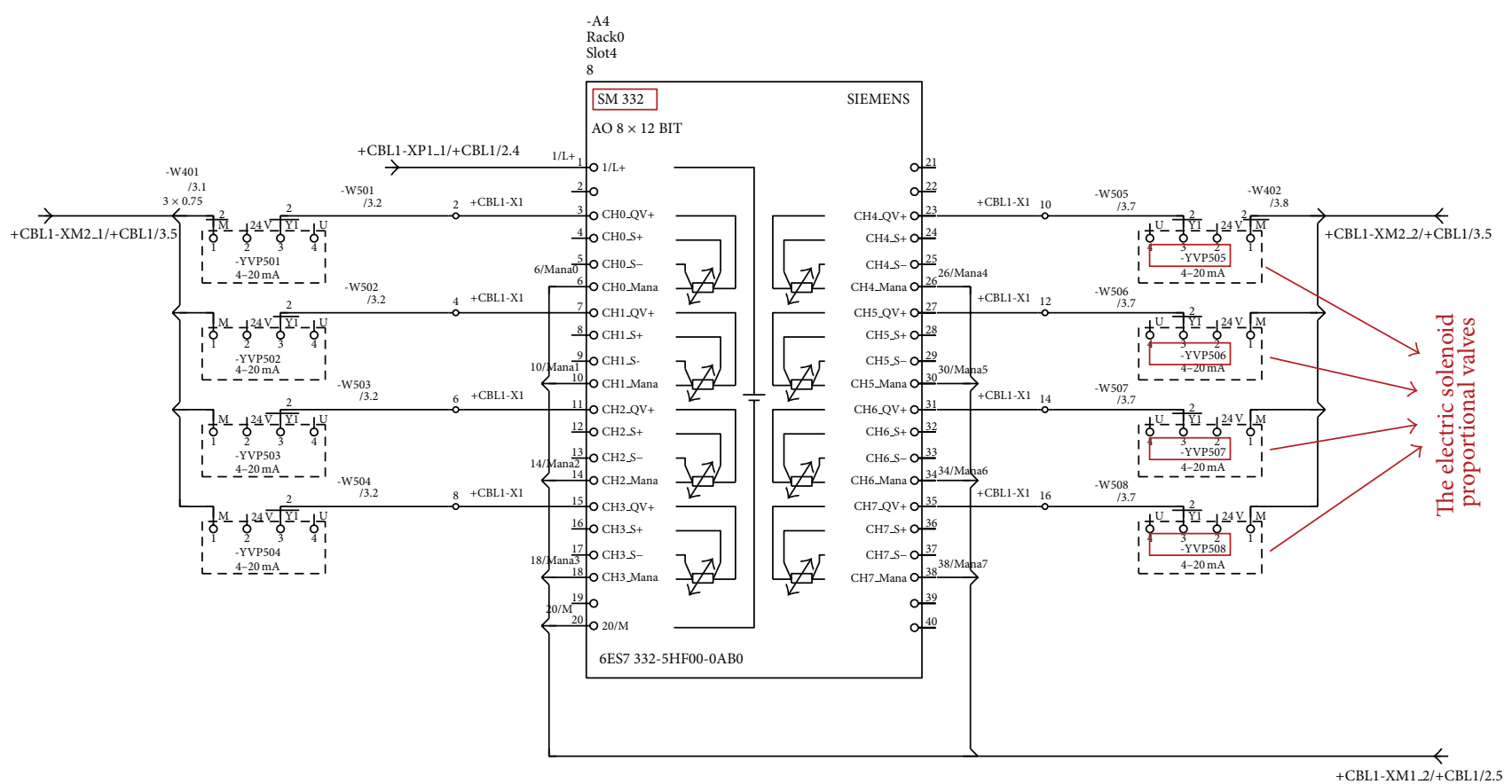

FIGURE 9: The circuit diagram for connecting the electric valves to the analog output module.

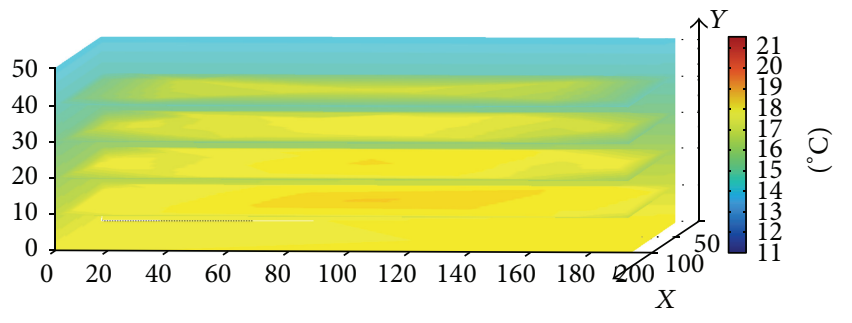

FIgURE 10: Real-time three-dimensional temperature field.

The proposed approach offers an effective and efficient alternative for acquiring temperature digital data useful for supporting the decision-making processes regarding the control of cooling of just poured mass concrete in real time.

A mathematical description of the digital temperature control model has been developed. Use of this model for temperature values acquisition, filtering, sorting, treating, and their real-time transfer for cooling control purposes is proved effective. A stable, real-time, high volume capacity of the temperature data transmission solution developed utilized an intelligent cooling control system.

Using this system on the Xiluodu arch dam site enabled some key problems to be solved including huge quantities of raw data interchange with the module server, personalized and flexible control for each water cooling circuit, and accurate and punctual water conditional control data collection instead of traditional manual checking. The proposed approach provides a convenient solution in process cooling control pipes where cabling is not possible. The system also has lower installation and maintenance costs, operates reliably, and is of robust and flexible construction. It is proved suitable for heat of hydration control in dam construction concreting applications.

Based on this study, the future research will be focused on the combination of real-time site monitoring analysis and numerical simulation on thermal stress for control cracking of mass concrete in construction period.

\section{Conflict of Interests}

The authors declare that there is no conflict of interests regarding the publication of this paper.

\section{Acknowledgments}

This research work was supported by National Natural Science Foundation of China (nos. 11272178 and 51339003), National Basic Research Program of China (973 Program) under Grants nos. 2013CB035902 and 2011CB013503, and Tsinghua University Initiative Scientific Research Program.

\section{References}

[1] P. Lin, Q. B. Li, S. W. Zhou, and Y. Hu, "Intelligent cooling control method and system for mass concrete," Journal of Hydraulic Engineering, vol. 44, no. 8, pp. 950-957, 2013.

[2] F. A. Branco and P. Mendes, "Numerical simulations of heat of hydration temperature in dams," in Proceedings of the International Workshop on Arch Dams, pp. 225-230, Coimbra, Portugal, April 1987.

[3] B. Zhu, "Effect of cooling by water flowing in nonmetal pipes embedded in mass concrete," Journal of Construction Engineering and Management, vol. 125, no. 1, pp. 61-68, 1999.

[4] A. M. Neville, Properties of Concrete, Longman, Essex, UK, 1995. 
[5] H. S. Shang, T. H. Yi, and Y. P. Song, "Behavior of plain concrete of a high water-cement ratio after freeze-thaw cycles," Materials, vol. 5, no. 9, pp. 1698-1707, 2012.

[6] H. S. Shang and T. H. Yi, "Freeze-thaw durability of airentrained concrete," The Scientific World Journal, vol. 2013, Article ID 650791, 6 pages, 2013.

[7] H. S. Shang and T. H. Yi, "Behavior of HPC with fly ash after elevated temperature," Advances in Materials Science and Engineering, vol. 2013, Article ID 478421, 7 pages, 2013.

[8] G. D. Zhou and T. H. Yi, "Thermal load in large-scale bridges: a state-of-the-art review," International Journal of Distributed Sensor Networks, vol. 2013, Article ID 217983, 17 pages, 2013.

[9] Q. Li, G. Li, and G. Wang, "Elasto-plastic bond mechanics of embedded fiber optic sensors in concrete under uniaxial tension with strain localization," Smart Materials and Structures, vol. 12, no. 6, pp. 851-858, 2003.

[10] P. Lin, Q. B. Li, and H. Hu, "A flexible network structure for temperature monitoring of a super high arch dam," International Journal of Distributed Sensor Networks, vol. 2012, Article ID 917849, 10 pages, 2012.

[11] Q. B. Li, P. Lin, S. W. Zhou et al., "Real time, online, individuation and intelligent cooling control system for mass concrete," Utility Model Patent, 2012204165226, 2013.

[12] P. Lin, Q. B. Li, Y. Hu et al., "A device of integrity flow and temperature controlling," Patent Number(s), 201220417714.9, 2013.

[13] Q. B. Li and P. Lin, "Demonstration on intelligent dam," Journal of Hydroelectric Engineering, vol. 33, no. 1, 2014.

[14] K. J. Astrom and T. Hagglund, PID Controllers: Theory, Design, and Tuning, International Society for Measurement and Control, Seattle, Wash, USA, 2nd edition, 1995.

[15] A. B. Corripio, Tuning of Industrial Control Systems, Instrument Society of America, Research Triangle Park, NC, USA, 1990.

[16] F. D. Petruzella, Programmable Logic Controllers, Mcgraw-Hill, New York, NY, USA, 3rd edition, 2004.

[17] M. Gopal, Digital Control and State Variable Methods: Conventional and Intelligent Control Systems, McGraw Hill Education, 4th edition, 2012.

[18] M. S. Santina, A. R. Stubberud, and G. H. Hostetter, Digital Control System Design, International Thomson Publishing, Stamford, Conn, USA, 2nd edition, 1994.

[19] F. Italiano, R. Maugeri, A. Mastrolia, and J. Heinicke, "A new seafloor monitoring module for real-time data transmission: an application to shallow hydrothermal vents," Procedia Earth and Planetary Science, vol. 4, pp. 93-98, 2011.

[20] R. Bayindir and Y. Cetinceviz, "A water pumping control system with a Programmable Logic Controller (PLC) and industrial wireless modules for industrial plants-an experimental setup," ISA Transactions, vol. 50, no. 2, pp. 321-328, 2011.

[21] J. Flores, B. Arcay, and J. Arias, "An intelligent system for distributed control of an anaerobic wastewater treatment process," Engineering Applications of Artificial Intelligence, vol. 13, no. 4, pp. 485-494, 2000.

[22] P. Mariño, M. Á. Domínguez, F. Poza, and F. Vázquez, “Using LOTOS in the specification of industrial bus communication protocols," Computer Networks, vol. 45, no. 6, pp. 767-799, 2004.

[23] J. L. Posadas, P. Pérez, J. E. Simó, G. Benet, and F. Blanes, "Communications structure for sensory data in mobile robots," Engineering Applications of Artificial Intelligence, vol. 15, no. 3-4, pp. 341-350, 2002.
[24] C. Hennig, T. Bluhm, P. Heimann et al., "Using the network as bus system for long discharge data acquisition and data processing," Fusion Engineering and Design, vol. 81, no. 15-17, pp. 17351740, 2006.

[25] R. Moraes, F. B. Carreiro, P. Bartolomeu, V. Silva, J. A. Fonseca, and F. Vasques, "Enforcing the timing behavior of real-time stations in legacy bus-based industrial Ethernet networks," Computer Standards and Interfaces, vol. 33, no. 3, pp. 249-261, 2011.

[26] C. Hennig, K. Kneupner, and D. Kinna, "Connecting Programmable Logic Controllers (PLC) to control and data acquisition a comparison of the JET and Wendelstein 7-X approach," Fusion Engineering and Design, vol. 87, no. 12, pp. 1972-1976, 2012.

[27] J.-N. Dong, G.-H. Qin, and H. Yu, "Study on real-time sensor signal transmission based on technology of polynomial predictive filtering," Measurement, vol. 42, no. 7, pp. 969-977, 2009.

[28] P. Lin, S. Z. Kang, Q. B. Li et al., "Evaluation on rock mass quality and stability of xiluodu arch dam under construction phase," Journal of Rock Mechanics and Engineering, vol. 31, no. 10, pp. 2042-2052, 2012.

[29] P. Lin, X. L. Liu, Y. Hu Yu et al., "Deformation and stability analysis on xiluodu arch dam under stress and seepage condition," Journal of Rock Mechanics and Engineering, vol. 32, no. 6, pp. 1137-1144, 2013. 


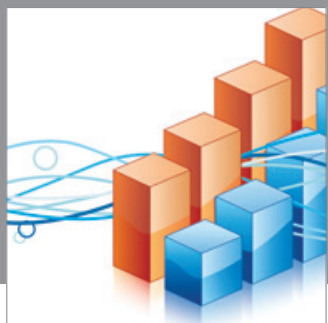

Advances in

Operations Research

mansans

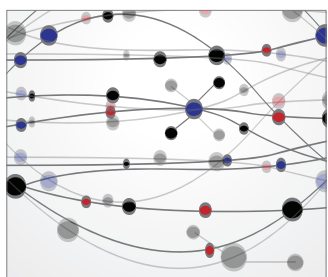

The Scientific World Journal
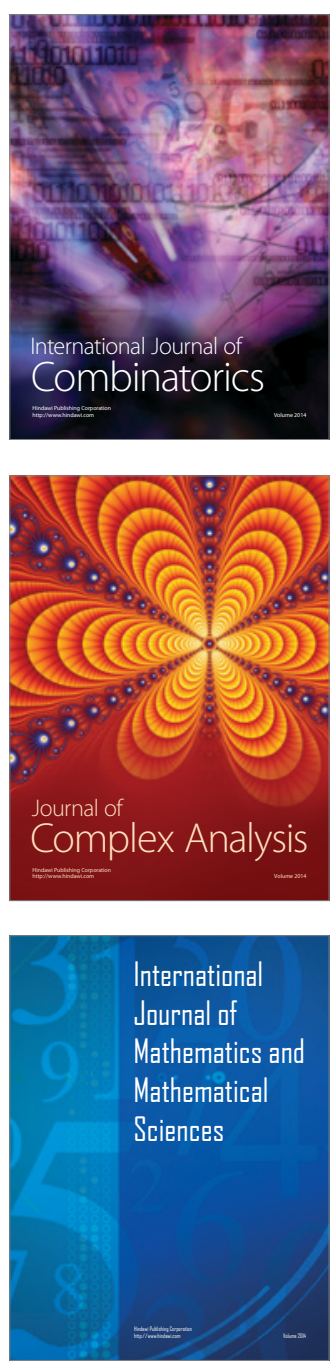
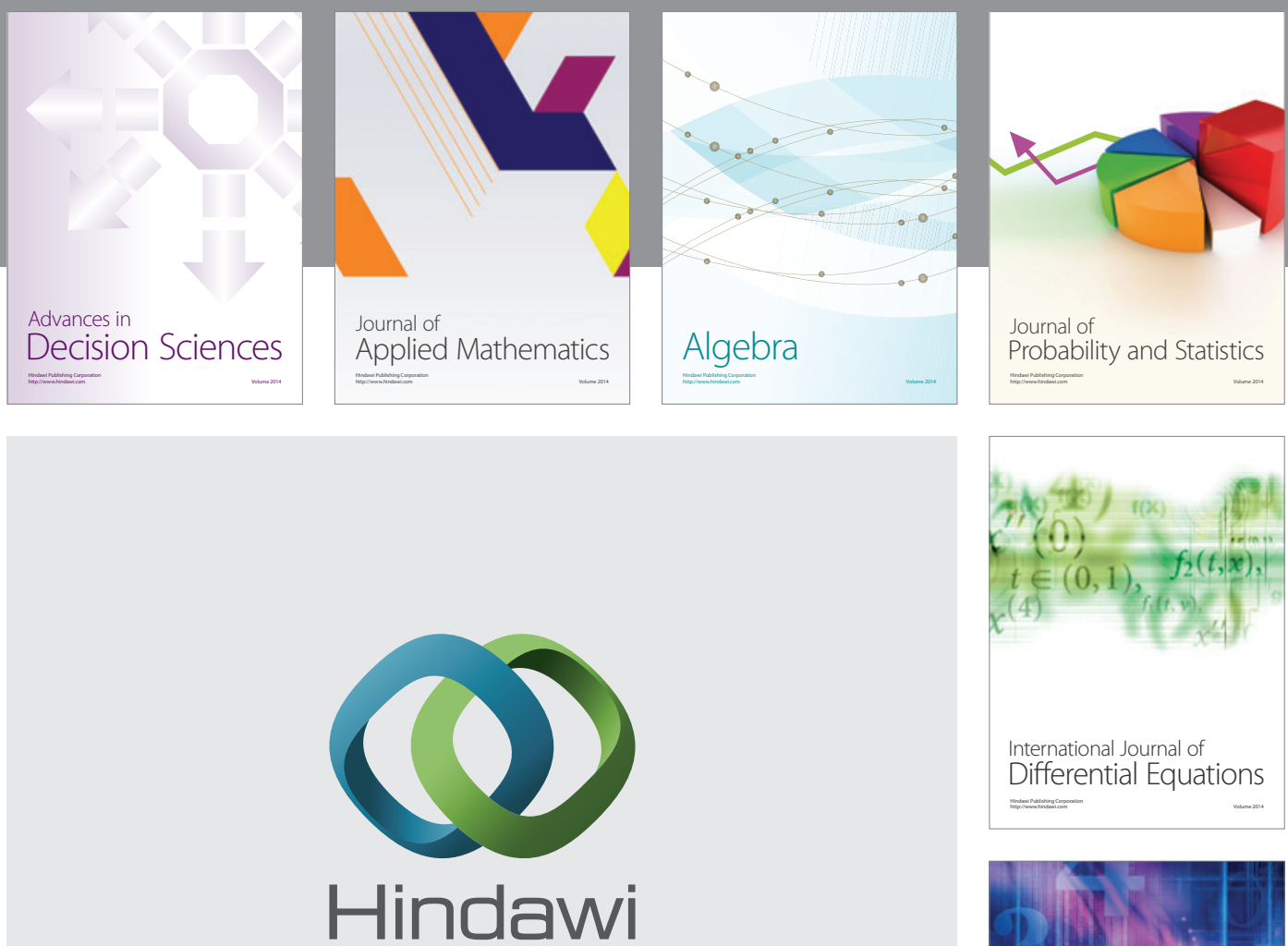

Submit your manuscripts at http://www.hindawi.com
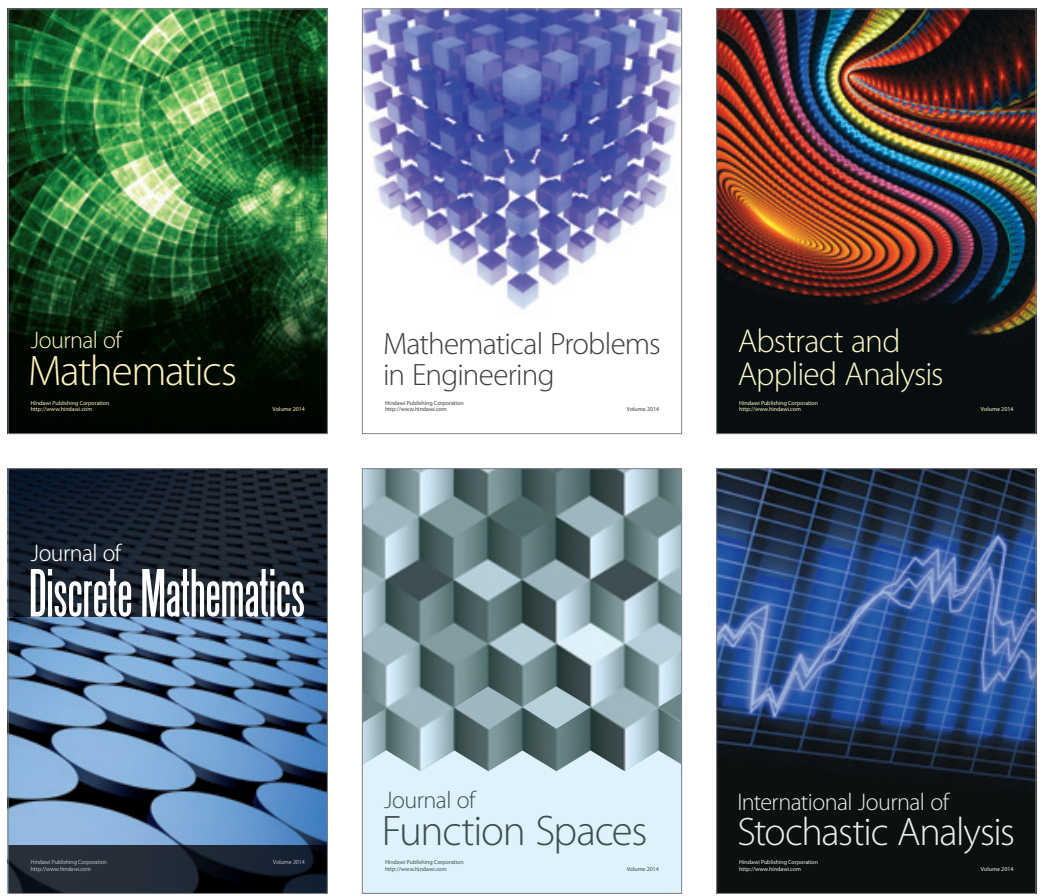

Journal of

Function Spaces

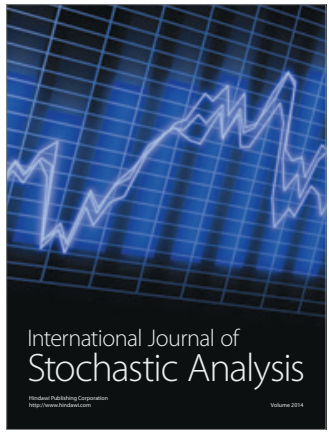

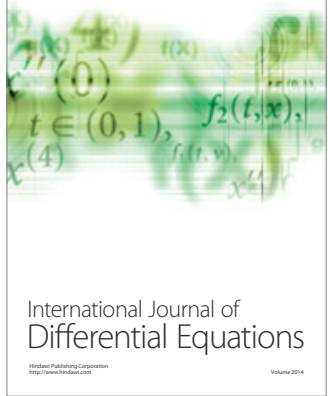
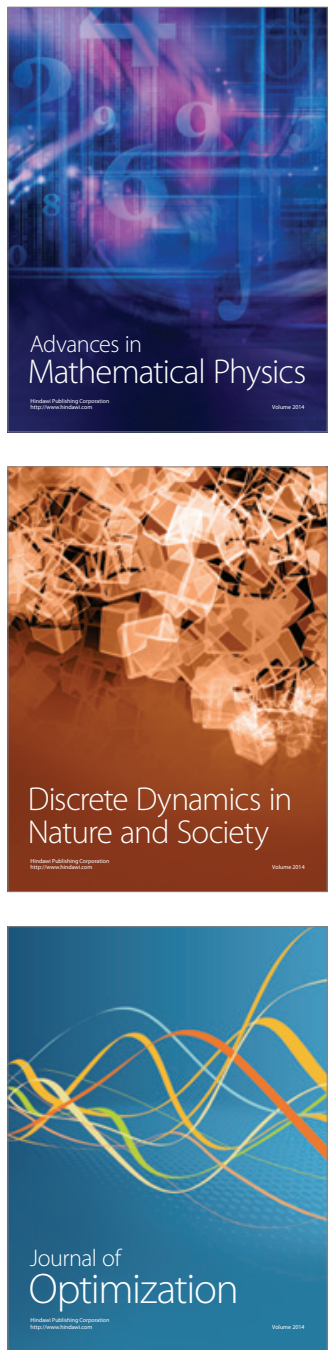